
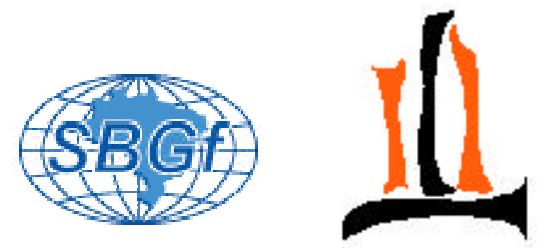

São Paulo 2004

\title{
Influência da Cobertura Vegetal Na Estrutura Térmica da Subsuperfície
}

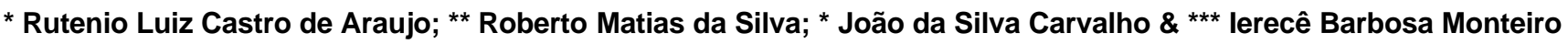 \\ * Universidade Federal do Amazonas; ** Fundação de Tecnologia do Estado do Acre; *** Universidade do Estado do \\ Amazonas
}

Copyright 2004, SBGf - Sociedade Brasileira de Geofísica

Este texto foi preparado para a apresentação no I Simpósio de Geofísica da Sociedade Brasileira de Geofísica, São Paulo, 26-28 de setembro de 2004. Seu conteúdo foi revisado pela Comissão Tecno-científica do I SR-SBGf mas não necessariamente representa a opinião da SBGf ou de seus associados. E proibida a reprodução total ou parcial deste material para propósitos comerciais sem prévia autorização da SBGf.

\section{Resumo}

Foi realizado, num período de um ano, monitoramento térmico diário às profundidades de $0,0 \mathrm{~m}$ e $1,0 \mathrm{~m}$, em dois locais contíguos, sendo um deles sem e outro com cobertura vegetal. Os resultados mostram variações mensuráveis dos valores da temperatura, sendo preponderantemente superiores aqueles relativos ao local sem cobertura vegetal. Tais resultados permitem a mensuração da influência da cobertura vegetal no regime geotermal raso, amplia e aprimora o conhecimento sobre a estrutura térmica das camadas subsurperficiais e, ainda, fornece dados imprescindíveis para a elaboração de modelos reais acerca dos efeitos dos processos de desmatamento e/ou reflorestamento na Amazônia. Registrou-se, também, a influência do parâmetro climatológico insolação.

\section{Introdução}

A estrutura geotermal rasa compreende toda a zona situada da superfície terrestre até a profundidade na qual são mensuráveis os efeitos termais provocados pela perturbação térmica gerada pela incidência da radiação solar nessa superfície terrestre, (Araujo, 1999). Assim sendo, o comportamento termal da estrutura geotermal rasa é cristalinamente influenciado por diversos parâmetros ambientais como, também, influencia vários deles (Souza et al., 1989; Araujo et al., 1991; Silva, 2003; Silva et al., 2003). Portanto, a fim de se ter um melhor conhecimento acerca da estrutura geotermal rasa, suas variações, magnitudes, fontes termais e seus efeitos, é de fundamental importância a execução de estudos atinentes à influência das fontes externas, principalmente no que concerne ao nível de cobertura vegetal, precipitação pluviométrica e insolação em uma determinada região.

Tais estudos propiciarão 0 aprimoramento do conhecimento dessa estrutura geotérmica rasa e, conseqüentemente, possibilitarão a elaboração de modelos reais os quais, além de descreverem o seu estado térmico atual, possam, também, prever resultados futuros.

\section{Materiais e Métodos}

Os trabalhos de pesquisa foram desenvolvidos na região de Manaus, em dois locais distintos, porém contíguos, sendo um sem cobertura vegetal (vegetação composta por esparsas gramas e arbustos, s/c) e outro com cobertura vegetal (vegetação composta por árvores de médio a grande porte, $\mathrm{c} / \mathrm{c}$ ). As pesquisas foram realizadas durante um ciclo climático completo, referente ao ano de 2000. As medidas de temperatura foram realizadas nos horários das $08 \mathrm{~h}$ e $17 \mathrm{~h}$, na superfície e à $1,0 \mathrm{~m}$ de profundidade.

As medidas de temperatura superficial foram realizadas com o emprego de um termômetro digital, tendo como sensor térmico um termopar (Robertson et al., 1966; Carvalho et al., 1986). A precisão dessas medidas foi de $\pm 0,01^{\circ} \mathrm{C}$. As medidas de temperatura a 0,5 e $1,0 \mathrm{~m}$ de profundidade foram realizadas utilizando-se um termômetro de termistor. A leitura da resistência elétrica do termistor foi obtida com o auxílio de uma ponte resistiva, tipo Wheatstone. A ponte resistiva foi adaptada para operar com um regulador de corrente contínua o que permitiu o ajuste da corrente aplicada em 20?A, durante todas as medidas. O baixo valor da corrente aplicada possibilita que a ponte permaneça ligada por algumas horas ininterruptas sem, entretanto, aquecer de forma detectável o sensor térmico. Este procedimento tornou possível a leitura da resistência elétrica do termistor com precisão de $\pm 2,0$ ? e resolução de $\pm 0,5$ ? . Os termistores foram previamente calibrados usando-se um termômetro de platina, com precisão de $\pm 0,001^{\circ} \mathrm{C}$. Desta forma, foi possível a elaboração de uma curva de calibração que permitiu a conversão dos valores medidos da resistência elétrica do termistor em valores precisos de temperatura. As variações da temperatura foram registradas com precisão de $\pm 0,01{ }^{\circ} \mathrm{C}$.

Os dados meteorológicos de precipitação pluviométrica e insolação foram obtidos junto ao $1^{\underline{\underline{0}}}$ Distrito de Meteorologia do Instituto Nacional de Meteorologia.

\section{Resultados e Discussões}

O Estado do Amazonas é caracterizado por apresentar, apenas, dois períodos distintos num ciclo climático completo: um período "chuvoso", dos meses de dezembro a maio e outro considerado "seco", dos meses de junho a novembro. Para fins de análises, consideramos os meses de abril e setembro como sendo os meses representativos dos períodos de maior e de menor precipitação pluviométrica, respectivamente.

As figuras 1 e 2 mostram os resultados dos valores diários medidos da temperatura superficial e da 
insolação, nos locais s/c e c/c, às 14:00h, referentes aos meses de abril e outubro, respectivamente. Nestas figuras observa-se, nitidamente, uma boa relação entre as configurações das curvas representativas dos valores da temperatura (para os locais s/c e c/c), com relação à curva referente à insolação. A somatória mensal da insolação teve o valor de $50,9 \mathrm{~h}$ no mês de abril e de $205,2 \mathrm{~h}$ no mês de outubro. A boa relação das variações dos parâmetros temperatura superficial e insolação caracteriza a insolação como uma eficiente e fundamental fonte externa a qual influencia, preponderantemente, os valores da temperatura às profundidades palco dos estudos da geotermia rasa. As figuras 1 e 2 mostram, também, de forma cristalina, a elevada superioridade dos valores da temperatura superficial registrados no local $\mathrm{s} / \mathrm{c}$, em relação aos valores obtidos no local c/c. O valor médio mensal da temperatura superficial no local $\mathrm{s} / \mathrm{c}$ foi de $31,13^{\circ} \mathrm{C}$ no mês de abril e de $32,68^{\circ} \mathrm{C}$ no mês de outubro. No local $\mathrm{c} / \mathrm{c}$ registrou-se o valor médio de $25,61^{\circ} \mathrm{C}$ no mês de abril e de $28,81^{\circ} \mathrm{C}$ no mês de outubro. Existe, então, uma diferença, entre as duas médias citadas, de $5,52^{\circ} \mathrm{C}$ no mês de abril e de $3,87^{\circ} \mathrm{C}$ no mês de outubro. Face ao fato de que os dois locais estarem contíguos e apresentarem litologias semelhantes ( $88 \%$ de argila), podemos concluir que a elevada variação nos valores médios mensais da temperatura superficial deve-se, exclusivamente, à diferença do grau de influência da cobertura vegetal. Assim sendo, mensurando tal influência torna-se possível, efetivamente, determinar a importância crucial da cobertura vegetal superficial sobre a estrutura geotermal rasa.

As figuras 3 e 4 apresentam os resultados dos valores medidos da temperatura à $1,0 \mathrm{~m}$ de profundidade e os de insolação, no horário das $14 \mathrm{~h}$, para os locais s/c e c/c, atinentes aos meses de abril e outubro, respectivamente. Nestas figuras verificam-se as mesmas boas relações entre os parâmetros envolvidos nas figuras 1 e 2, como também, a nítida superioridade dos valores registrados da temperatura à $1,0 \mathrm{~m}$ de profundidade no local s/c em relação ao local c/c. A temperatura média mensal à $1,0 \mathrm{~m}$ de profundidade no local s/c foi de $25,11^{\circ} \mathrm{C}$ no mês de abril e de $26,99^{\circ} \mathrm{C}$ no mês de outubro; enquanto que no local c/c registrou-se um valor médio desse parâmetro da ordem de $24,62^{\circ} \mathrm{C}$ no mês de abril e de $24,76^{\circ} \mathrm{C}$ no mês de outubro. $\mathrm{O}$ que denota uma variação da temperatura média mensal, à $1,0 \mathrm{~m}$ de profundidade, de $0,49^{\circ} \mathrm{C}$ no mês de abril e de $2,23^{\circ} \mathrm{C}$ no mês de outubro.

Como comprobação da influência do efeito do fluxo de radiação solar que incide na superfície terrestre, como um agente externo atuante sobre o regime geotermal raso, apresentamos as figuras 5 e 6 . A figura 5 mostra os valores da temperatura média mensal e da insolação para o ano de 2000 , às 14:00h, em superfície, nos locais $\mathrm{s} / \mathrm{c}$ e c/c; enquanto que a figura 6 apresenta os mesmos parâmetros da figura 5 , sendo que os registros da temperatura correspondem a 1,0m de profundidade.

Desta forma, os resultados supra apresentados mostram a irrefutável importância das fontes externas sobre o regime geotermal raso, em especial, no que se refere à cobertura vegetal, por ser este filtro um dos eficientes gerenciadores das variações térmicas subsuperficiais.
Tais resultados fornecem, também, a mensuração dos valores da temperatura em locais com e sem cobertura vegetal, os quais são fundamentais para a elaboração de modelos reais que almejam explicar as grandes mudanças globais e, ainda, efetivar previsões concernentes, tendo como base as variações térmicas provocadas pelo processo de desmatamento.

\section{Conclusões}

A análise dos resultados do presente trabalho embasa as seguintes conclusões:

No período e locais estudados ocorrem variações consideráveis dos valores da temperatura, tanto em superfície como a $1,0 \mathrm{~m}$ de profundidade, as quais não podem ser consideradas como negligenciáveis;

Durante todo o ciclo climático de um ano, os valores diurnos como, também, as médias mensais da temperatura superficial e à $1,0 \mathrm{~m}$ de profundidade foram mensuravelmente superiores no local sem cobertura vegetal, em relação ao local com cobertura vegetal. A maior diferença entre os valores diurnos da temperatura superficial, do local s/c em relação ao local $\mathrm{c} / \mathrm{c}$, foi registrado no dia 4 de outubro, às $14: 00 \mathrm{~h}$, cuja magnitude foi de $7,20^{\circ} \mathrm{C}$. A $1,0 \mathrm{~m}$ de profundidade, esta diferença foi de $2,47^{\circ} \mathrm{C}$ em data de 6 de outubro. Em relação aos valores das médias mensais da temperatura, a maior diferença registrada entre os locais $\mathrm{s} / \mathrm{c}$ e $\mathrm{c} / \mathrm{c}$ foi de $8,59^{\circ} \mathrm{C}$, à profundidade $0,0 \mathrm{~m}$ e de $2,57^{\circ} \mathrm{C}$ à profundidade de $1,0 \mathrm{~m}$; ambas foram registradas no mês de setembro. Há, portanto, mensurável variação nos valores diurnos e nas médias mensais da temperatura. Tais variações são decorrentes, fundamentalmente, do tipo de cobertura vegetal superficial;

Há uma boa relação entre as variações dos parâmetros temperatura, tanto superficial quanto à $1,0 \mathrm{~m}$ de profundidade, com as mudanças do índice de insolação. Isto, independentemente da profundidade e locais estudados, caracterizando, assim, uma relação diretamente proporcional entre as variações dos parâmetros insolação e temperatura.

Os resultados obtidos no presente trabalho permitem mensurar a influência da cobertura vegetal superficial no estado térmico das camadas subsuperficiais, as quais constituem-se palco da geotermia rasa. Tais valores são de suma importância na elaboração de modelos reais acerca dos efeitos causados pelos processos de desmatamento ou de reflorestamento na Amazônia.

\section{Agradecimentos}

Externamos nossos sinceros agradecimentos; $\grave{A}$ Universidade Federal do Amazonas, à Universidade do Estado do Amazonas e à Fundação de Tecnologia do Estado do Acre, por permitirem aos autores dedicação à pesquisa. Ao Instituto Nacional de Meteorologia - INMET, $1^{0}$ Distrito de Meteorologia, pelo fornecimento dos dados de precipitação pluviométrica e insolação. 


\section{Referências Bibliográficas}

ARAUJO, R. L. C. 1999. Contribuição da Geotermia Rasa aos Estudos Ambientais. Manaus. Ed. Universidade do Amazonas. 88p.

ARAUJO, R. L. C.; SOUZA, J. R. S. e MAKINO, M. 1991. Análise de perfis geotérmicos rasos em poços sujeitos a aquecimento superficial e bombeamento de água. Revista Brasileira de Geofísica. 9: 47 - 53.

ARAUJO, R.L.C.; CARVALHO, J.S. e FERNANDES L.S. 1991. A condutividade térmica em solos argilosos em Manaus. Anais do III Simpósio de Geologia da Amazônia. Belém - PA. 350 - 360 .

CARVALHO, H. S.; LOBO, P. F. S.; CAMPOS, J. N. P. and ZEMBRUSCKI, S. G. 1986. Heat Flow and Hydrodinamic Study in the Medium Amazon Basin. Im: International Meeting on Geothermics and Geothermal Energy. São Paulo - SP, agosto, 107 - 110.

ROBERTSON, E. C.; RASPET, R.; SWARTZ, J. H. and LIILARD, M. E. 1966. Properties of Thermistors Used in Geothermal Investigations. Geological Survey Bulletin. 1203-B: 1-34.

SILVA, R.M. 2003. Influencia Ambiental Sobre a Estrutura Geotermal Rasa. Dissertação de mestrado. Dezembro. 102.p.

SILVA, R.M.; ARAUJO. R.L.C. e CARVALHO, J.S. 2003. Variação Temporal da Temperatura. Anais do $8^{0}$ Congresso Internacional da Sociedade Brasileira de Geofísica. Rio de Janeiro - RJ, setembro. 236 - 242.

SOUZA, J. R. S.; ARAUJO, R. L. C. e MAKINO, M. 1989. Heat Transfer and Thermal Properties of the Subsoil in Belém. Revista Brasileira de Geofísica. 7 : 19-28.

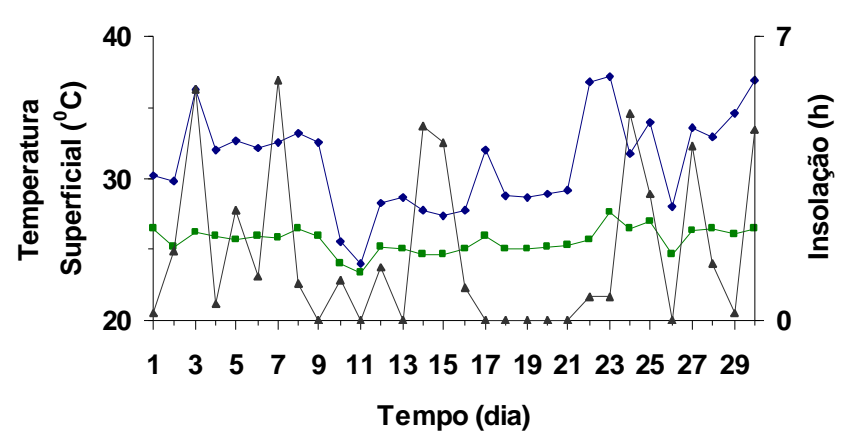

$\rightarrow$ s/c $\rightarrow$ c/c $\rightarrow$ Insolação

FIGURA 1 - Valores diários da temperatura superficial e da insolação, referentes ao mês de abril/2000, às 14:00h, nos locais s/c e c/c.

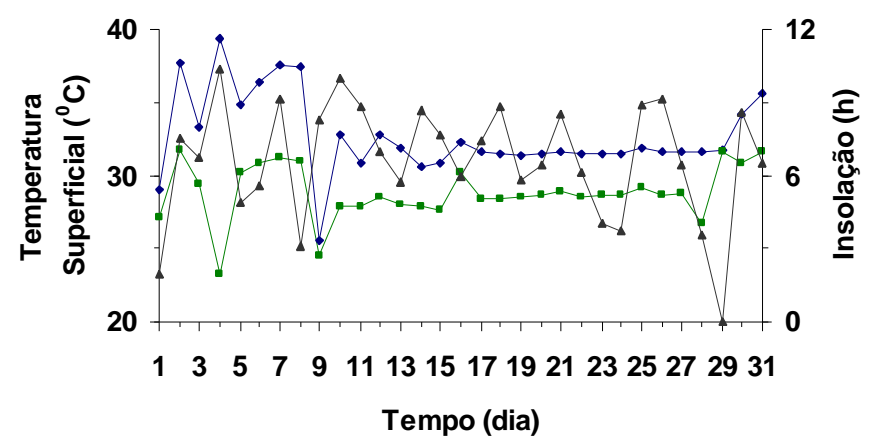

$\bullet$ s/c $-\mathrm{c} / \mathrm{c} \rightarrow$ Insolação

FIGURA 2 - Valores diários da temperatura superficial e da insolação, referentes ao mês de outubro/2000, às 14:00h, nos locais s/c e c/c. 


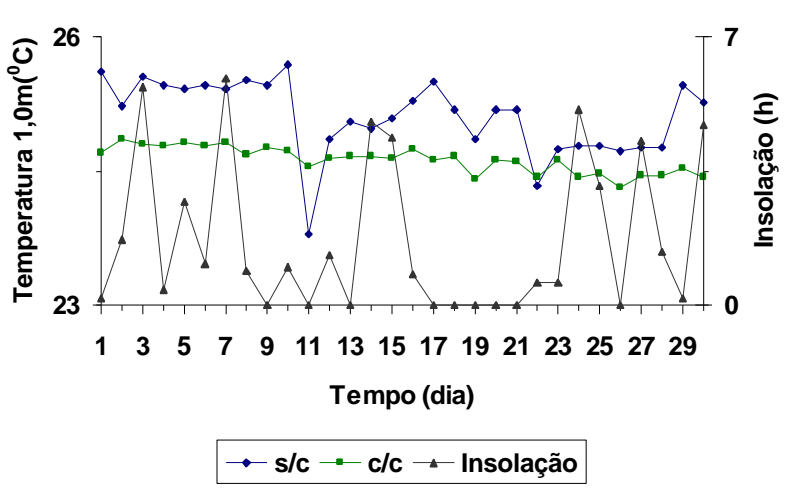

FIGURA 3 - Valores diários da temperatura a 1,0m de profundidade e da insolação, referentes ao mês de abril/2000, às 14:00h, nos locais, s/c e c/c.

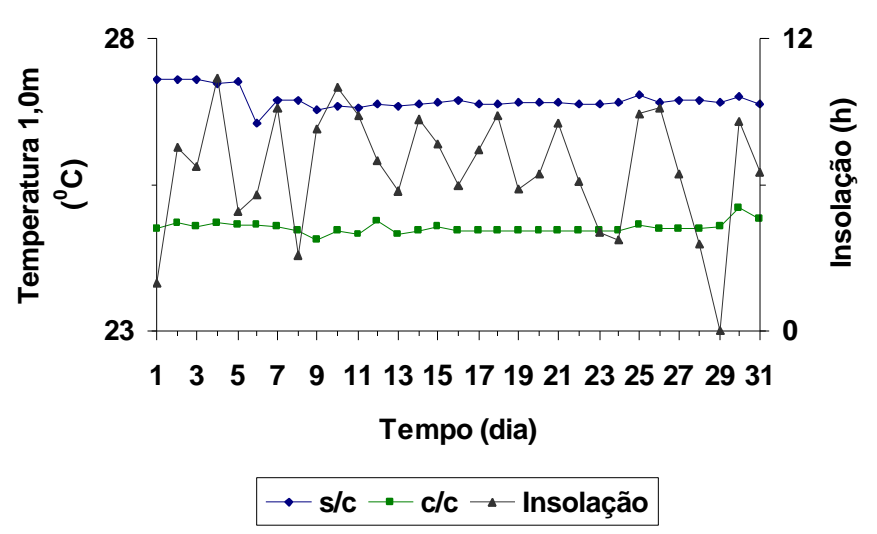

FIGURA 4 - Valores diários da temperatura à 1,0m de profundidade e da insolação, referentes ao mês de outubro/2000, às 14:00h, nos locais, s/c e c/c.

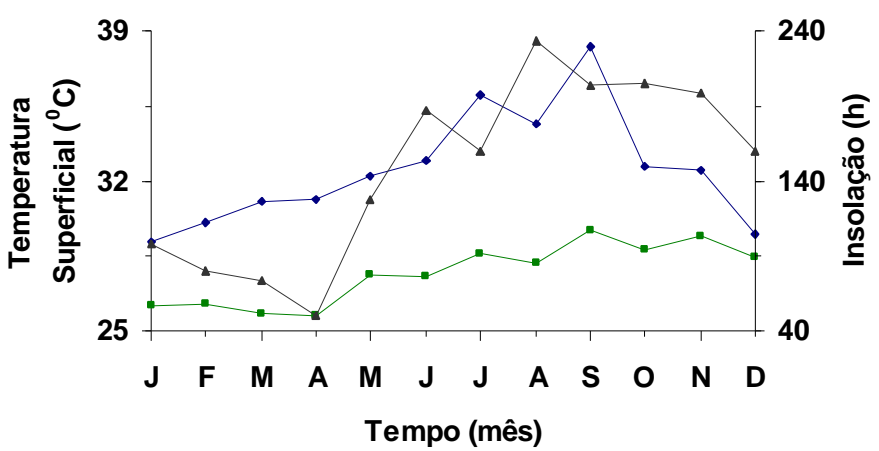

$$
\bullet \mathbf{s} / \mathbf{c} \rightarrow \mathbf{c} / \mathbf{c} \rightarrow \text { Insolação }
$$

FIGURA 5 - Valores médios mensais da temperatura superficial e da insolação, referentes ao ano de 2000, às 14:00h, nos locais s/c e c/c.

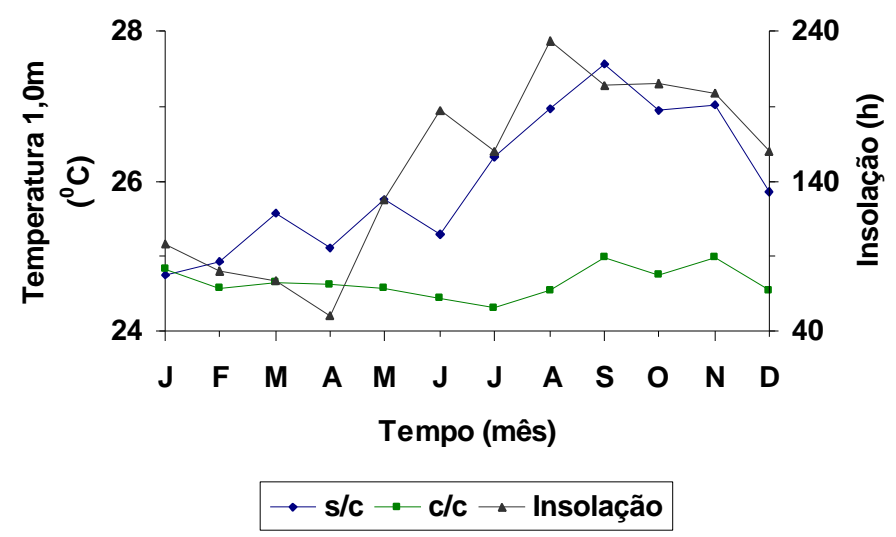

FIGURA 6 - Valores médios mensais da temperatura à $1,0 \mathrm{~m}$ de profundidade e da insolação, referentes ao ano de 2000, às 14:00h, nos locais, s/c e c/c. 